\title{
Accelerating Ovarian Age: Cancer Treatment in the Premenopausal Woman
}

\author{
Joseph Letourneau, MD ${ }^{1,2} \quad$ S. W. Chan, BS ${ }^{1} \quad$ Mitchell P. Rosen, MD, HCLD ${ }^{1}$ \\ ${ }^{1}$ Division of Reproductive Endocrinology, Department of Obstetrics, \\ Gynecology, and Reproductive Sciences, University of California, San \\ Francisco, California \\ 2 Department of Obstetrics and Gynecology, University of North \\ Carolina, Chapel Hill, North Carolina

\begin{abstract}
Address for correspondence Mitchell P. Rosen, MD, HCLD, Department of Obstetrics, Gynecology, and Reproductive Sciences, UCSF, 1635 Divisadero Street, Suite 601, San Francisco, CA 94115 (e-mail: rosenm@obgyn.ucsf.edu).
\end{abstract}

Semin Reprod Med 2013;31:462-468

\begin{abstract}
Keywords

- cancer

- ovarian reserve

- ovarian age

- menopause

- ovarian failure

- chemotherapy

- radiation

The reproductive sequelae of cancer treatments may provide an important model of accelerated ovarian aging. Tens of thousands of women treated for cancer each year experience infertility and early menopause as a result of treatment. A spectrum of reproductive compromise commonly ranges from immediate menopause at the time of cancer treatment to the less proximate outcome of early menopause in the years to decades after treatment. A woman's reproductive lifespan can be shortened after chemotherapy or radiation because such treatments likely decrease the number of viable eggs after treatment. This acceleration in the decline of the number of follicles leads to increased rates of not only infertility and miscarriage but also early menopause, which represents the most extreme form of accelerated ovarian aging. The degree of reproductive impairment is dependent on chronologic age and the diagnosis or treatment. The variation in outcomes that persist may be partially explained by pretreatment ovarian reserve. Establishing the use of clinical predictors such as ovarian reserve markers to effectively anticipate such outcomes is an obvious and important keystone in the foundation of cancer survivorship research. An improved understanding of cancer treatment's ability to accelerate follicle death, decrease fecundability, and initiate an earlier menopause could provide a clinically relevant, time-shortened, and reproducible snapshot into the basic biology of ovarian aging.
\end{abstract}

According to 2011 Surveillance Epidemiology and End Results, 123,000 young adult women ages 18 to 44 are diagnosed with cancer every year in the United States. ${ }^{1,2}$ Furthermore, an additional 6,000 children and adolescents are treated for cancer each year., ${ }^{3,4}$ Many of these children and young adult women will face adverse reproductive outcomes after treatment. ${ }^{5-8}$ Such diminished reproductive potential after cancer treatment can negatively impact quality of life in young survivors. ${ }^{8-10}$ From a patient perspective, studies have indicated that the potential iatrogenic loss of fertility may have as profound impact on young women as the cancer diagnosis itself. ${ }^{11}$
A woman's reproductive lifespan can be shortened after chemotherapy or radiation because such treatments likely decrease the number of viable eggs after treatment. ${ }^{6,12}$ In this article, we will review: the reproductive impact of cancer treatment and the clinical risk factors associated with such impact. To conclude, we will discuss treatment-associated infertility and early menopause as a model of accelerated ovarian aging. An improved understanding of cancer treatment's ability to accelerate follicle death, decrease fecundability, and initiate an earlier menopause could provide a clinically relevant, time-shortened, and reproducible snapshot into the basic biology of ovarian aging.

Copyright $\odot 2013$ by Thieme Medical Publishers, Inc., 333 Seventh Avenue, New York, NY 10001, USA. Tel: +1(212) 584-4662.
DOI http://dx.doi.org/ $10.1055 / \mathrm{s}-0033-1356482$. ISSN 1526-8004.
Issue Theme Diminished Ovarian Reser
Guest Editor, Marcelle I. Cedars, MD 


\section{Cancer Treatment as an Accelerator of Ovarian Age}

Our understanding of how cancer treatment may affect ovarian age continues to evolve. ${ }^{12-14}$ In the past, when assessing the gonadotoxicity of cancer treatments, most studies used the presence or absence of normal menses as their primary outcome. ${ }^{15,16}$ This approach had limitations in terms of improving our understanding of fertility and premature menopause as outcomes remote from treatment. Albright et al first described primary ovarian insufficiency (also known as premature ovarian failure) in $1942^{17,18}$; since then an increased understanding has been gained regarding partial ovarian injury. Primary ovarian insufficiency can be overt (menses are absent or irregular) or occult (the ovary is damaged but menses remain regular). ${ }^{19}$ Clinically, young patients treated for cancer may notice a complete loss of menstrual function in the weeks to months around treatment. ${ }^{18,20}$ However, many will sustain partial ovarian injury, which may manifest not as immediate loss of menses, but as infertility or early menopause. $12,21,22$

A key proposed mechanism for such injury to the ovary is that cancer treatments lead to apoptotic oocyte death in growing follicles, which accelerates more follicles to come out of the quiescent state, and subsequently be lost to further apoptotic cell death. ${ }^{12,21,23,24}$ The number of follicles remaining in the ovaries has potential implications for predicting reproductive outcomes. One such implication may be that as follicle counts (ovarian reserve) diminish with age, the quality of oocytes also declines, resulting in an increased incidence of infertility and miscarriage rates. ${ }^{25-27}$ As a result, in patients that are exposed to cancer treatment, acute follicle loss can result in accelerated reproductive decline. ${ }^{28}$ The potential outcomes of such accelerated decline can range from subtle changes such as infertility or early menopause in the years to decades after treatment is completed to the most severe: amenorrhea in the weeks to months after chemotherapy or radiation.

\section{Reproductive Compromise after Treatment: A Problem of Significant Scale}

Most of the aims of research about reproductive health outcomes after cancer treatment have focused on amenorrhea as a primary outcome and have been focused on breast cancer. When focusing on breast cancer alone, it appears that as few as $40 \%$ of women continue to menstruate after treatment. ${ }^{1-3,5-10,19,22,29-34}$ When other cancer types are included, however, a higher proportion of women continue to menstruate after treatment. For example, two-thirds of women from the California Cancer Registry who were treated with chemotherapy alone for nongynecologic malignancies noted a continuation of menses posttreatment. ${ }^{15,29,35}$ And, in recently submitted data from our group, $57 \%$ of women with cervical or uterine cancers and $93 \%$ with ovarian cancers in a California Cancer Registry survey reported the presence of menses after fertility sparing surgery for localized disease. In both of these groups, the persistence of menses is more

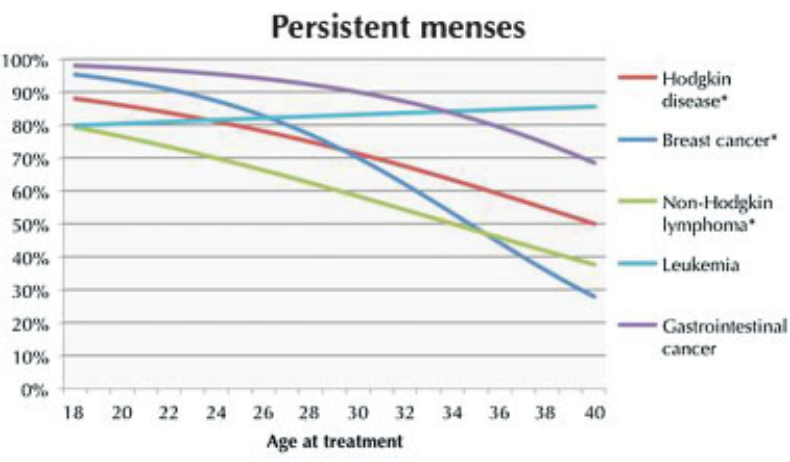

Figure 1 With advancing age, the chance of persistent menses decreases after exposure to chemotherapy $\left({ }^{*} p<0.05\right.$, Hodgkin disease, breast cancer, non-Hodgkin lymphoma). Adapted from Letourneau et al. ${ }^{29}$

common among women who are younger at the time of treatment ( $\mathbf{F i g} \mathbf{1} \mathbf{1})$.

Menses may return to women who became amenorrheic temporarily. One-third of women noted cessation of menses after treatment with chemotherapy alone for nongynecologic cancers. However, half of these women noted a subsequent return of menses. Similar rates of subsequent return of onceabsent menses were noted in a recent review by Amir et al. $8,19,22,29,30,34,36$

As few as 10 to $20 \%$ of women ages 18 to 40 who were treated with chemotherapy alone report a permanent loss of menses shortly after treatment. Among the remaining 80 to $90 \%$ who have menses, however, elevated proportions of infertility and early menopause were noted remote from treatment, suggesting a role for accelerated ovarian aging as a mediator of their reproductive health outcomes. Women who had menses at some point after treatment for leukemia, Hodgkin disease, non-Hodgkin lymphoma, breast cancer, and gastrointestinal (GI) malignancies were noted to be at a substantially increased risk of infertility over the background rate in the United States. ${ }^{6,7,10,20,22,29,31,33,37}$ Such women were also noted to have an age-dependent elevation in risk of early menopause in the years to decades after treatment (-Fig. 2).

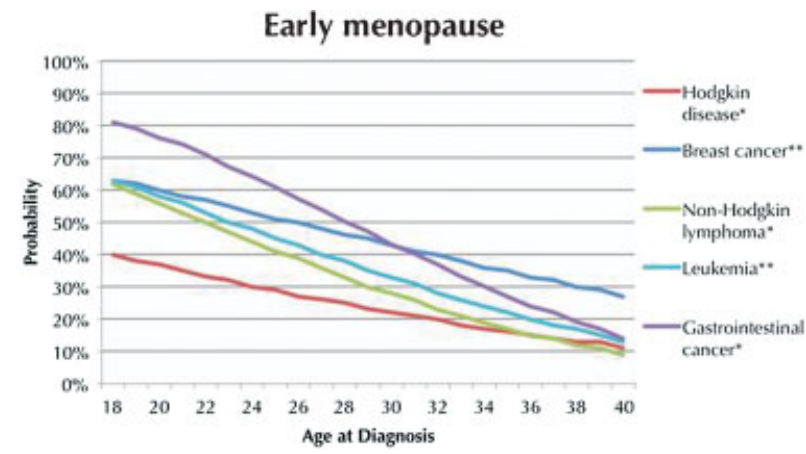

Figure 2 With advancing age, the probability of early menopause decreases after exposure to chemotherapy $\left({ }^{*} p<0.05\right.$, Hodgkin disease, non-Hodgkin lymphoma, gastrointestinal cancer; ${ }^{* *} p<0.01$, breast cancer, leukemia). Adapted from Letourneau et al. ${ }^{22}$ 


\section{Early Menopause}

Those women whose menses continue despite treatment for cancer face an increased risk of early menopause (menses before 45 years old) in the years to decades after treatment. In survivors of childhood cancers, the risk of developing nonsurgical premature menopause was 13-fold higher than that of siblings, with a cumulative incidence of $8 \%$ by age 40 years. $^{23}$ of the numerous young adult women in the United States expected to menstruate at some point after completion of cancer treatment, between 10 and $90 \%$ may go on to experience early menopause. The wide variance in outcomes may be influenced by ovarian reserve, genetic susceptibility, age at treatment, time elapsed since treatment, and treatment type. $22,30,38-41$

Multiple studies have demonstrated women that have menses posttreatment who are younger in age have a higher probability of chemotherapy-induced early menopause in the years to decades after treatment. ${ }^{22,38}$ - Fig. 2 illustrates agespecific rates of early menopause in women treated with chemotherapy for various disease types in women who had menstruated after treatment: Hodgkin disease, non-Hodgkin lymphoma, and GI malignancies. For instance, 18-year-old women with non-Hodgkin lymphoma are at a fivefold increased risk of experiencing early menopause versus their 40year-old counterparts. ${ }^{22}$ Unpublished data from the California Cancer Registry also demonstrates a significant relationship between younger age at treatment and probability of early menopause in women treated with fertility sparing surgery and chemotherapy for gynecologic cancers who note menses in the weeks to months posttreatment. Such results argue for the importance of counseling younger women about their risk of a shortened reproductive window.

It has been suggested that temporary amenorrhea in the posttreatment period could be associated with a higher risk of early menopause than if menses persisted without temporary cessation. Reports have been conflicting, with some studies suggesting an increased probability of early menopause after temporary amenorrhea whereas others have not. ${ }^{29,38}$ This discrepancy could represent a difference in ovarian response based on treatment type, the age of the study population, or the duration of posttreatment amenorrhea that is noted (-Fig. 3) ${ }^{29}$ In addition, the differences in genetic variance and ovarian reserve amongst those women who note temporary amenorrhea versus those who do not are presently not well understood. ${ }^{41}$

Among women who have menses present after cancer therapy, recent data suggest that infertility in the posttreatment period likely portends to a higher risk for subsequent early menopause. ${ }^{29}$ In healthy women, fecundity generally decreases drastically and somewhat predictably approximately 10 years before the average age of menopause (51 years old) (-Fig. 4). 2,3,9,15,22,29,35 Women who are still menstruating but who have a history of posttreatment infertility should be made aware that their probability of entering menopause before the age of 45 is approximately twofold higher (vs. if they had been able to conceive posttreatment). ${ }^{29}$ This observation should not be surprising given that other studies in the general population have shown that, while

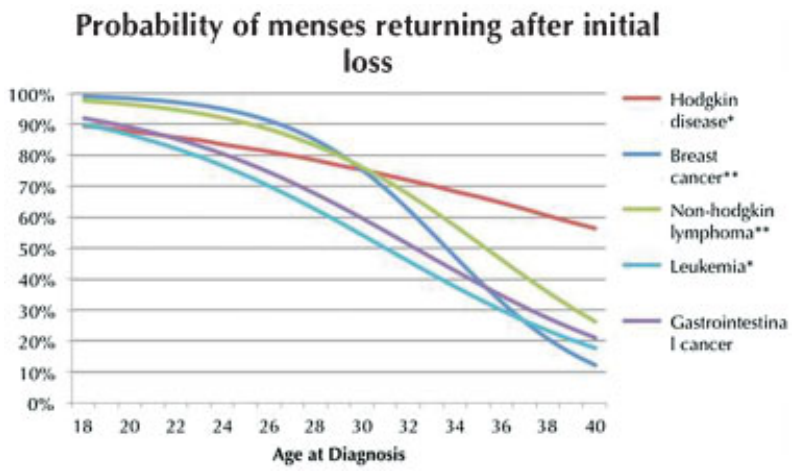

Figure 3 With advancing age, the chance of return of menses once amenorrheic decreases after exposure to chemotherapy $\left({ }^{*} p<0.05\right.$, Hodgkin disease, leukemia; ${ }^{* *} p<0.01$, breast cancer, non-Hodgkin lymphoma). Adapted from Letourneau et al. ${ }^{29}$

menses are still present, subfertility often represents the first manifestation of incipient ovarian failure. $8,11,22,29$

Along with the difficult consequences of infertility, loss of ovarian function in patients who are survivors of cancer has a profound impact on their hormonal milieu and potentially their subsequent risk for the development of chronic disease. ${ }^{42}$ Establishing the use of clinical predictors to effectively anticipate such outcomes is an obvious and important keystone in the foundation of cancer survivorship research.

Recent studies have shown that measuring ovarian reserve markers before treatment may provide further guidance into predicting the degree of reproductive compromise after treatment. Evidence has shown that lower antral follicle count (AFC) and/or anti-Mullerian hormone (AMH) are associated with a higher incidence of posttreatment amenorrhea (-Fig. 5). ${ }^{43}$ These observations suggest that women who have advanced ovarian age before treatment are more likely to experience reproductive compromise regardless of chronologic age. ${ }^{28}$ This, along with genetic variance, may explain some of the variation in reproductive impairment that we observe for a given treatment and chronologic age. ${ }^{40}$ Other studies have shown markers of ovarian reserve decline at different rates depending on the gonadotoxicity of the regimen (-Fig. 6). ${ }^{44}$ Decanter et al published their preliminary

\section{Onset of Infertility Linked to Onset of Menopause}

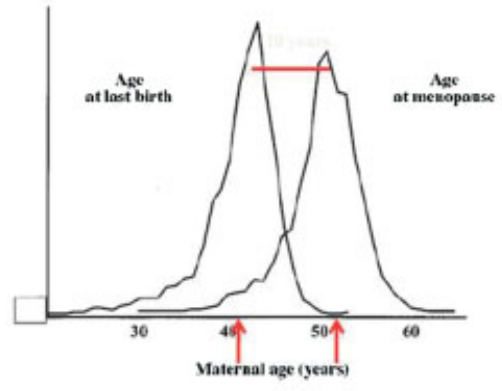

TeVelde et 2l, 2002

Figure 4 In healthy women, fecundity generally decreases approximately 10 years before the average age of menopause (51 years old) (adapted from TeVelde et al. The variability of female redproductive ageing. Hum Reprod Update 2002;8(2):141-154. Used with permission. ${ }^{62}$ ). 

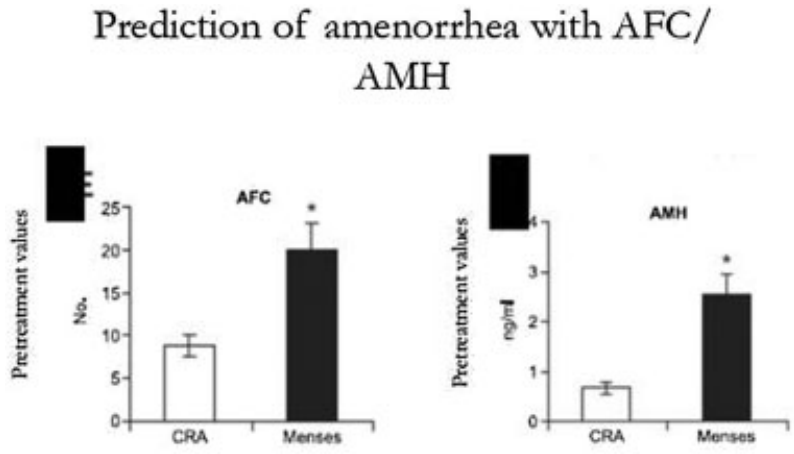

Anderson, et al, 2011

Figure 5 Lower AFC and AMH are associated with posttreatment amenorrhea. AFC, antral follicle count; $\mathrm{AMH}$, anti-Mullerian hormone. (Adapted from Anderson and Cameron. ${ }^{43}$ )

results showing how AMH levels change with exposure to chemotherapy and up to 12 months thereafter in patients exposed to Adriamycin, Bleomycin, Vinblastine, Dacarbazine (ABVD) and non-ABVD regimens. ${ }^{44}$ AMH levels decrease during chemotherapy and can rebound thereafter. ${ }^{44,45}$ They observed the rebound in ovarian reserve markers is more dramatic with ABVD, which suggests this regimen is less toxic to the nongrowing pool of follicles. These findings correlate with the higher incidence of temporary amenorrhea with the ABVD regimens, compared with non-ABVD regimens that we observe. ${ }^{44}$ Other studies have shown that higher doses of alkylating agents have been correlated with decreased AMH levels. As the gonadotoxicity of therapy increases, AMH levels approach those of perimenopausal women ( - Fig. 7). ${ }^{46}$ These data support that $\mathrm{AMH}$ can detect differences in ovarian toxicity between chemotherapy regimens. In addition, AFC has been shown to be decrease in women who are survivors of childhood cancer treatment, despite continuation of menses, compared with controls that were not treated for cancer. ${ }^{46}$ These observations are not surprising given that ovarian

\section{AMH follow -up after chemotherapy}

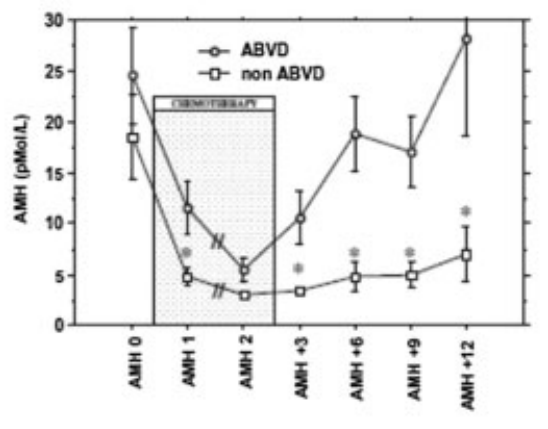

Decanter, 2009

Figure $6 A B V D$ and non-ABVD regimens are shown to affect $A M H$ levels differently during the first 12 months after chemotherapy (adapted from Decanter et al. Anti-mullerian hormone follow-up in young women treated by chemotherapy for lymphoma preliminary results. Reprod Biomed Online 2010;20(2):280-285. Used with permission. ${ }^{63}$ ).

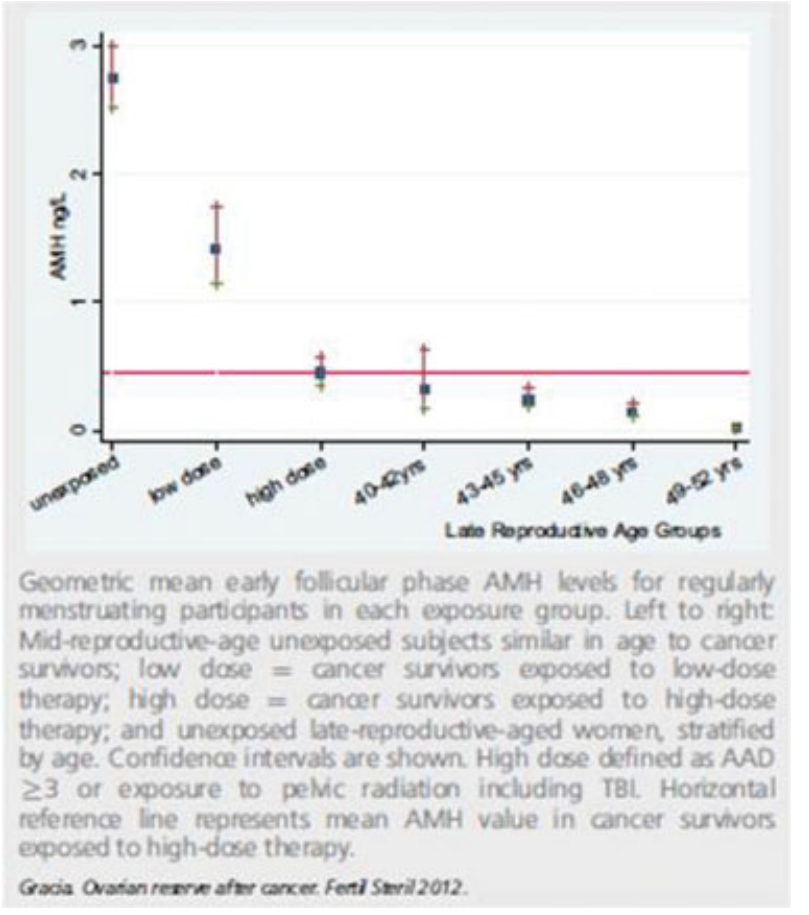

Figure 7 In increasingly gonadotoxic cancer therapies, AMH levels approach those of perimenopausal women (adapted from Garcia et al. Impact of cancer therapies on ovarian reserve. Fertil Steril 2012;97(1): 134-140.e1. Used with Permission. ${ }^{64}$ ).

reserve markers are an estimate of the number of nongrowing follicles that are remaining in the ovary. ${ }^{47-49}$

\section{Infertility and Early Pregnancy Loss}

Among women with menses present after treatment, infertility rates in the years and decades after treatment are elevated as high as two to four times the background rate in the United States. ${ }^{10,22,33}$ Overall percentages of 12-month infertility among women who continued to menstruate after treatment with chemotherapy alone were $20,18,15,27$, and $23 \%$ for women in the California Cancer Registry with a history leukemia, Hodgkin disease, non-Hodgkin lymphoma, breast cancer, and GI malignancies, respectively. ${ }^{22}$ Similar rates of infertility were seen in the Norwegian Cancer Registry, in which $25 \%$ of 184 women with similar time to followup for Hodgkin disease and attempting conception were unable to conceive. ${ }^{32}$ Studies that have reported on postdiagnosis pregnancy rates, rather than infertility, report pregnancy rates from 9 to $13 \%$ for breast cancer survivors. ${ }^{50-52}$ Although low pregnancy rates can be influenced by many factors including age, fear of recurrence, delayed attempts at conception, treatment-related sexual dysfunction, and diagnosis-related relationship instability, a major factor is the permanent impact of adjuvant systemic therapies on ovarian function and reserve. ${ }^{13,21,53}$

Women who experience temporary amenorrhea report rates of infertility that may be doubled versus those whose periods continued through chemotherapy. ${ }^{29}$ Data from the California Cancer Registry suggest that the absence of menses during or after their treatment is associated with a further loss of future reproductive capacity. ${ }^{29}$ In other words, a 
temporary chemotherapy-induced amenorrhea may correlate with an independent increase in risk of infertility or reproductive compromise. Partridge et al noted a similar association in a prospective cohort study utilizing International Breast Study Group data. ${ }^{38}$

When pregnancy does occur, the risk of spontaneous miscarriage may be elevated in those with a history of systemic chemotherapy exposure. ${ }^{54}$ Recently submitted data from our group also show miscarriage rates in the California Cancer Registry which were significantly higher among women with pregnancies after treatment for earlystage gynecologic cancers that underwent fertility sparing surgery than they were for nongynecologic cancers, despite controlling for pelvic radiation. This increased rate of early pregnancy loss after cancer treatment further supports the notion that treatment is associated with advancement of ovarian age. Prior studies have demonstrated an association between decreased ovarian reserve and higher rates of miscarriage. ${ }^{25-27}$

Older chronologic age at the time of treatment has been associated with an increased risk of infertility after chemotherapy (-Fig. 8). This is likely related to age-related depletion of ovarian reserve before initiation of cancer therapy. However, an association between ovarian reserve markers and infertility and miscarriage has yet to be clearly established amongst a diverse group of cancers. Nonetheless, it is reasonable to assume that such a relationship exists given the accelerated loss in follicle count that results from the chemotherapy and/or radiation. An ability to better predict increased risk for reproductive difficulties after treatment could improve counseling for the 123,000 reproductive-age women diagnosed with cancer each year in the United States. ${ }^{55-57}$

\section{Immediate Menopause}

A minority of women treated for cancer will not see advanced ovarian aging manifest in a time period remote from treatment. Approximately 10 to $30 \%$ of those women treated for cancer during their reproductive years will manifest the most severe form of reproductive compromise-immediate meno-

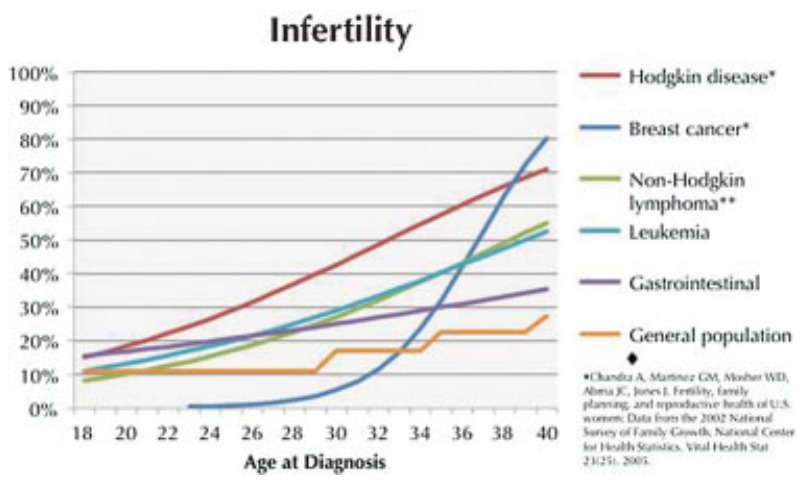

Figure 8 With advancing age, the incidence of infertility increases after exposure to chemotherapy at a greater rate than the general United States population $\left({ }^{*} p<0.05\right.$, Hodgkin disease, breast cancer; ${ }^{* *} p<0.01$, nonHodgkin lymphoma). Adapted from Letourneau et al. ${ }^{29}$ pause amenorrhea. ${ }^{22}$ This form of ovarian injury is manifest by relatively few women who are treated with chemotherapy alone, but is much more prevalent amongst women being treated with bone marrow transplant or pelvic radiation. Among breast cancer patients in the California Cancer Registry, for example, $9 \%$ experience acute loss of menses after treatment and no return within 1 year. Similarly, approximately 7 to $8 \%$ of women treated with chemotherapy alone for Hodgkin disease noted permanent cessation of menses. ${ }^{22,58}$ Taken together, women in the United States who manifest permanent amenorrhea at the time of cancer treatment comprise tens of thousands of patients who experience a similarly acute and devastating loss of ovarian function.

Radiation and various chemotherapies each differ in their mechanism of cancer cell cytotoxicity or cell cycle inhibition and, consequently, given treatment types exert varied effects on ovarian function. External beam radiation induces widespread DNA injury via free radical production within the radiated field. Alkylating agents (cyclophosphamide/temozolomide) lead to cross-linking of DNA strands in cell nuclei with relatively little dependence on active cell division for their cytotoxic effect. Each of these modalities presumably cause more widespread ovarian damage as they significant effect DNA stability in both growing and quiescent follicles. ${ }^{14}$ Platinum-containing compounds (cisplatin/carboplatin/oxaliplatin) may act similarly in the ovary, via DNA cross-linking. Agents that act via cell cycle inhibition such as topoisomerase inhibitors or anthracyclines (topotecan/doxorubicin), as well as antimetabolites (methotrexate/fluorouracil), likely exert a more selective effect on growing follicles. Over $80 \%$ of women treated with external beam pelvic radiation or bone marrow transplant will subsequently experience amenorrhea. Alkylating agents, particularly the combination regimen of oral cyclophosphamide, methotrexate, and fluorouracil), are associated with the highest risk of ovarian failure in patients with breast cancer, reaching $90 \%$ in women older than 40 years. ${ }^{3}$ Conversely, regimens such as Adriamycin, vinblastine, bleomycin, dacarbazine) for Hodgkin disease have been thought to exert less gonadotoxicity, with reported acute ovarian failure rates often less than $20 \%{ }^{14}$ This risk of amenorrhea also increases with higher cumulative doses of chemotherapy, particularly with alkylating drugs and when using taxanes and anthracyclines in combination. ${ }^{30,36,59,60}$ As one ages, smaller and smaller doses are needed to produce ovarian failure. ${ }^{61}$ This is consistent with the observation that women with lower ovarian reserve markers before treatment are more likely to experience posttreatment amenorrhea. ${ }^{44,47}$

\section{Treatment-Associated Infertility and Early Menopause Model Accelerated Ovarian Aging}

Tens of thousands of women treated for cancer each year will go on to experience infertility and early menopause as a result of treatment-induced advancement of their ovarian age. This has been demonstrated in several ways. Women exposed to chemotherapy have lower ovarian reserve than expected for their age group. Variability in ovarian reserve may help 
account for the observed variation in rates of ovarian insufficiency in age-matched individuals who have undergone cancer treatment. Women that receive more toxic treatments have lower ovarian reserve markers. These ovarian reserve markers are correlated with the number of eggs that are remaining in the ovary. This acceleration in the decline of the number of follicles leads to increased rates of not only infertility but also early menopause, which represents the most extreme form of accelerated ovarian aging.

In Western societies during the past 50 years, increasing educational level and participation in the labor force have been associated with a rise in the mean age at which women deliver their first child. Female fertility starts to decline from the early 20s because of decreasing ovarian reserve-a term that refers to both the quality and the quantity of the ovarian follicle pool. Consequently, many women will be faced with unexpected fertility problems as a result of their ovarian age. Women with advancing ovarian age must too face the potential health consequences associated with the menopausal transition, including osteoporosis and cardiovascular disease.

Given the tremendous cost burden on our health care system of infertility, osteoporosis, and cardiovascular disease, efforts made to mitigate their frequency should be lauded by health policy makers. Unfortunately, under average circumstances, these outcomes and the factors (such as advancing ovarian age) that are associated with them take years to decades to manifest and are therefore difficult to study. An improved understanding of the clinical consequences and translational biology of ovarian aging provides an opportunity to make an immeasurable impact on health care costs and outcomes. We have demonstrated in this article how cancer treatment's ability to accelerate follicle death, decrease fecundability, and initiate an earlier menopause in tens of thousands of women can provide a clinically relevant, time-shortened, and reproducible snapshot into the basic biology of ovarian aging.

\section{References}

1 Siegel R, Naishadham D, Jemal A. Cancer statistics, 2013. CA Cancer J Clin 2013;63(1):11-30

2 Siegel R, DeSantis C, Virgo K, et al. Cancer treatment and survivorship statistics, 2012. CA Cancer J Clin 2012;62(4):220-241

3 Bines J, Oleske DM, Cobleigh MA. Ovarian function in premenopausal women treated with adjuvant chemotherapy for breast cancer. J Clin Oncol 1996;14(5):1718-1729

4 Chemaitilly W, Mertens AC, Mitby P, et al. Acute ovarian failure in the childhood cancer survivor study. J Clin Endocrinol Metab 2006;91(5):1723-1728

5 Warne GL, Fairley KF, Hobbs JB, Martin FI. Cyclophosphamideinduced ovarian failure. N Engl J Med 1973;289(22):1159-1162

6 Meirow D. Reproduction post-chemotherapy in young cancer patients. Mol Cell Endocrinol 2000;169(1-2):123-131

7 Knobf MT. The influence of endocrine effects of adjuvant therapy on quality of life outcomes in younger breast cancer survivors. Oncologist 2006;11(2):96-110

8 Letourneau JM, Ebbel EE, Katz PP, et al. Pretreatment fertility counseling and fertility preservation improve quality of life in reproductive age women with cancer. Cancer 2012;118(6): $1710-1717$
9 Patel A, Sreedevi M, Malapati R, et al. Reproductive health assessment for women with cancer: a pilot study. Am J Obstet Gynecol 2009;201(2):e1-e4

10 Tschudin S, Bitzer J. Psychological aspects of fertility preservation in men and women affected by cancer and other life-threatening diseases. Hum Reprod Update 2009;15(5):587-597

11 Carter J, Rowland K, Chi D, et al. Gynecologic cancer treatment and the impact of cancer-related infertility. Gynecol Oncol 2005; 97(1):90-95

12 Wallace WHB. Oncofertility and preservation of reproductive capacity in children and young adults. Cancer 2011;117(10, Suppl):2301-2310

13 Anderson RA, Wallace WHB. Antimüllerian hormone, the assessment of the ovarian reserve, and the reproductive outcome of the young patient with cancer. Fertil Steril 2013;99(6):1469-1475

14 Lee SJ, Schover LR, Partridge AH, et al; American Society of Clinical Oncology. American Society of Clinical Oncology recommendations on fertility preservation in cancer patients. J Clin Oncol 2006;24(18):2917-2931

15 Rugo HS, Rosen MP. Reducing the long-term effects of chemotherapy in young women with early-stage breast cancer. JAMA 2011;306(3):312-314

16 Schover LR. Patient attitudes toward fertility preservation. Pediatr Blood Cancer 2009;53(2):281-284

17 Albright F, Smith PH, Fraser R. A syndrome characterized by primary ovarian insufficiency and decreased stature: report of 11 cases with a digression on hormonal control of axillary and pubic hair. Am J Med Sci 1942;204:625-648

18 Letourneau JM, Melisko ME, Cedars MI, Rosen MP. A changing perspective: improving access to fertility preservation. Nat Rev Clin Oncol 2011;8(1):56-60

19 Nelson LM. Clinical practice. Primary ovarian insufficiency. N Engl J Med 2009;360(6):606-614

20 Chemaitilly W, Mertens AC, Mitby P, et al. Acute ovarian failure in the childhood cancer survivor study. J Clin Endocrinol Metab 2006;91(5):1723-1728

21 Morgan S, Anderson RA, Gourley C, Wallace WH, Spears N. How do chemotherapeutic agents damage the ovary? Hum Reprod Update 2012;18(5):525-535

22 Letourneau JM, Ebbel EE, Katz PP, et al. Acute ovarian failure underestimates age-specific reproductive impairment for young women undergoing chemotherapy for cancer. Cancer 2012; 118(7):1933-1939

23 Sklar CA, Mertens AC, Mitby P, et al. Premature menopause in survivors of childhood cancer: a report from the childhood cancer survivor study. J Natl Cancer Inst 2006;98(13):890-896

24 Rosen MP, Sternfeld B, Schuh-Huerta SM, Reijo Pera RA, McCulloch CE, Cedars MI. Antral follicle count: absence of significant midlife decline. Fertil Steril 2010;94(6):2182-2185

25 Rosen MP, Johnstone E, Addauan-Andersen C, Cedars MI. A lower antral follicle count is associated with infertility. Fertil Steril 2011;95(6):1950-1954, e1

26 Levi AJ, Raynault MF, Bergh PA, Drews MR, Miller BT, Scott RT Jr. Reproductive outcome in patients with diminished ovarian reserve. Fertil Steril 2001;76(4):666-669

27 Elter K, Kavak ZN, Gokaslan H, Pekin T. Antral follicle assessment after down-regulation may be a useful tool for predicting pregnancy loss in in vitro fertilization pregnancies. Gynecol Endocrinol 2005;21(1):33-37

28 Rosendahl M, Andersen CY, la Cour Freiesleben N, Juul A, Løssl K, Andersen AN. Dynamics and mechanisms of chemotherapy-induced ovarian follicular depletion in women of fertile age. Fertil Steril 2010;94(1):156-166

29 Letourneau JM, Niemasik EE, McCulloch CE, et al. Temporary amenorrhea predicts future infertility in young women treated with chemotherapy. Journal of Cancer Theraputics \& Research 2013;2:1-9 
30 Petrek JA, Naughton MJ, Case LD, et al. Incidence, time course, and determinants of menstrual bleeding after breast cancer treatment: a prospective study. J Clin Oncol 2006;24(7):1045-1051

31 Sukumvanich P, Case LD, Van Zee K, et al. Incidence and time course of bleeding after long-term amenorrhea after breast cancer treatment: a prospective study. Cancer 2010;116(13):3102-3111

32 Kiserud CE, Fosså A, Holte H, Fosså SD. Post-treatment parenthood in Hodgkin's lymphoma survivors. Br J Cancer 2007;96(9): 1442-1449

33 Forman EJ, Anders CK, Behera MA. A nationwide survey of oncologists regarding treatment-related infertility and fertility preservation in female cancer patients. Fertil Steril 2010;94(5): 1652-1656

34 Loprinzi CL, Wolf SL, Barton DL, Laack NN. Symptom management in premenopausal patients with breast cancer. Lancet Oncol 2008;9(10):993-1001

35 Sonmezer M, Oktay K. Fertility preservation in female patients. Hum Reprod Update 2004;10(3):251-266

36 Amir E, Freedman O, Allen L, Colgan T, Clemons M. Defining ovarian failure in amenorrheic young breast cancer patients. Breast 2010;19(6):545-548

37 Di Cosimo S, Alimonti A, Ferretti G, et al. Incidence of chemotherapy-induced amenorrhea depending on the timing of treatment by menstrual cycle phase in women with early breast cancer. Ann Oncol 2004;15(7):1065-1071

38 Partridge A, Gelber S, Gelber RD, Castiglione-Gertsch M, Goldhirsch A, Winer E. Age of menopause among women who remain premenopausal following treatment for early breast cancer: longterm results from International Breast Cancer Study Group Trials V and VI. Eur J Cancer 2007;43(11):1646-1653

39 Luciano AM, Franciosi F, Lodde V, et al. Oocytes isolated from dairy cows with reduced ovarian reserve have a high frequency of aneuploidy and alterations in the localization of progesterone receptor membrane component 1 and aurora kinase B. Biol Reprod 2013;88(3):58

40 Schuh-Huerta SM, Johnson NA, Rosen MP, Sternfeld B, Cedars MI, Reijo Pera RA. Genetic markers of ovarian follicle number and menopause in women of multiple ethnicities. Hum Genet 2012;131(11):1709-1724

41 van Dorp W, van den Heuvel-Eibrink MM, Stolk L, et al. Genetic variation may modify ovarian reserve in female childhood cancer survivors. Hum Reprod 2013;28(4):1069-1076

42 De Vos M, Devroey P, Fauser BCJM. Primary ovarian insufficiency. Lancet 2010;376(9744):911-921

43 Anderson RA, Cameron DA. Pretreatment serum anti-müllerian hormone predicts long-term ovarian function and bone mass after chemotherapy for early breast cancer. J Clin Endocrinol Metab 2011;96(5):1336-1343

44 Decanter C, Morschhauser F, Pigny P, Lefebvre C, Gallo C, Dewailly D. Anti-Müllerian hormone follow-up in young women treated by chemotherapy for lymphoma: preliminary results. Reprod Biomed Online 2010;20(2):280-285

45 Brougham MFH, Crofton PM, Johnson EJ, Evans N, Anderson RA, Wallace WH. Anti-Müllerian hormone is a marker of gonadotoxicity in pre- and postpubertal girls treated for cancer: a prospective study. J Clin Endocrinol Metab 2012;97(6):2059-2067

46 Gracia CR, Sammel MD, Freeman E, et al. Impact of cancer therapies on ovarian reserve. Fertil Steril 2012;97(1):134-140, e1

47 Grynnerup AG-A, Lindhard A, Sørensen S. The role of anti-Müllerian hormone in female fertility and infertility - an overview. Acta Obstet Gynecol Scand 2012;91(11):1252-1260
48 Kevenaar ME, Meerasahib MF, Kramer P, et al. Serum anti-mullerian hormone levels reflect the size of the primordial follicle pool in mice. Endocrinology 2006;147(7):3228-3234

49 Appt SE, Clarkson TB, Chen H, et al. Serum antimüllerian hormone predicts ovarian reserve in a monkey model. Menopause 2009;16(3):597-601

50 Velentgas P, Daling JR, Malone KE, et al. Pregnancy after breast carcinoma: outcomes and influence on mortality. Cancer 1999;85(11):2424-2432

51 Rippy EE, Karat IF, Kissin MW. Pregnancy after breast cancer: the importance of active counselling and planning. Breast 2009;18(6): 345-350

52 Overbeek A, van den Berg MH, Kremer LC, et al; DCOG LATER-VEVO study group. A nationwide study on reproductive function, ovarian reserve, and risk of premature menopause in female survivors of childhood cancer: design and methodological challenges. BMC Cancer 2012;12:363

53 Reulen RC, Zeegers MP, Wallace WH, et al; British Childhood Cancer Survivor Study. Pregnancy outcomes among adult survivors of childhood cancer in the British Childhood Cancer Survivor Study. Cancer Epidemiol Biomarkers Prev 2009;18(8):2239-2247

54 Blakely LJ, Buzdar AU, Lozada JA, et al. Effects of pregnancy after treatment for breast carcinoma on survival and risk of recurrence. Cancer 2004;100(3):465-469

55 Schover LR, Brey K, Lichtin A, Lipshultz LI, Jeha S. Knowledge and experience regarding cancer, infertility, and sperm banking in younger male survivors. J Clin Oncol 2002;20(7):1880-1889

56 Schover LR. Motivation for parenthood after cancer: a review. J Natl Cancer Inst Monogr 2005;2005(34):2-5

57 Niemasik EE, Letourneau J, Dohan D, et al. Patient perceptions of reproductive health counseling at the time of cancer diagnosis: a qualitative study of female California cancer survivors. J Cancer Surviv 2012;6(3):324-332

58 Behringer K, Breuer K, Reineke T, et al; German Hodgkin's Lymphoma Study Group. Secondary amenorrhea after Hodgkin's lymphoma is influenced by age at treatment, stage of disease, chemotherapy regimen, and the use of oral contraceptives during therapy: a report from the German Hodgkin's Lymphoma Study Group. J Clin Oncol 2005;23(30):7555-7564

59 Anderson RA, Themmen APN, Al-Qahtani A, Groome NP, Cameron DA. The effects of chemotherapy and long-term gonadotrophin suppression on the ovarian reserve in premenopausal women with breast cancer. Hum Reprod 2006;21(10):2583-2592

60 Tham Y-L, Sexton K, Weiss H, Elledge R, Friedman LC, Kramer R. The rates of chemotherapy-induced amenorrhea in patients treated with adjuvant doxorubicin and cyclophosphamide followed by a taxane. Am J Clin Oncol 2007;30(2):126-132

61 Whitehead E, Shalet SM, Blackledge G, Todd I, Crowther D, Beardwell CG. The effect of combination chemotherapy on ovarian function in women treated for Hodgkin's disease. Cancer 1983; 52(6):988-993

62 TeVelde E, Pearson P. The variability of female reproductive ageing. Hum Reprod Update 2002;8(2):141-154

63 Decanter C, Morschnauser F, Pigny P, et al. Anti-mullerian hormone follow-up in young women treated by chemotherapy for lymphoma preliminary results. Reprod Biomed Online 2010; 20(2):280-285

64 Garcia C, Samuel M, Freeman E, et al. Impact of cancer therapies on ovarian reserve. Fertil Steril 2012;97(1):134-140. e1 